\title{
Cadmium Increases the Sensitivity of Adolescent Female Mice to Nicotine-Related Behavioral Deficits
}

\author{
Philip Adeyemi Adeniyi, ${ }^{1}$ Babawale Peter Olatunji, ${ }^{2}$ Azeez Olakunle Ishola, ${ }^{3}$ \\ Duyilemi Chris Ajonijebu, ${ }^{4}$ and Olalekan Michael Ogundele ${ }^{1}$ \\ ${ }^{1}$ Department of Anatomy, Cell Biology and Neuroscience Unit, College of Medicine and Health Sciences, College Building II, \\ Afe Babalola University, Room G14, KM 8.5 Afe Babalola Way, PMB 5454, Ado-Ekiti, Ekiti State, Nigeria \\ ${ }^{2}$ Department of Biological Sciences, College of Sciences, Afe Babalola University, Ado Ekiti, Nigeria \\ ${ }^{3}$ Department of Anatomy, College of Health Sciences, University of Ilorin, Ilorin, Nigeria \\ ${ }^{4}$ Department of Physiology, College of Medicine and Health Sciences, Afe Babalola University, Ado Ekiti, Nigeria
}

Correspondence should be addressed to Olalekan Michael Ogundele; mikealslaw@hotmail.com

Received 31 August 2014; Accepted 22 October 2014; Published 16 November 2014

Academic Editor: Antonio Pisani

Copyright (C) 2014 Philip Adeyemi Adeniyi et al. This is an open access article distributed under the Creative Commons Attribution License, which permits unrestricted use, distribution, and reproduction in any medium, provided the original work is properly cited.

\begin{abstract}
This study investigates spatial and nonspatial working memory, anxiety related behavior, and motor activities in cadmium and/or nicotine exposed female adolescent mice. P28 female adolescent mice (albino strain) were divided into four groups of five $(n=5)$ mice each. A set of mice (Nic) received subcutaneous nicotine $(2.0 \mathrm{mg} / \mathrm{kg})$ while a separate set $(\mathrm{Cd})$ was treated with $2.0 \mathrm{mg} / \mathrm{kg}$ cadmium (subcutaneous). For the combined treatments of cadmium and nicotine, we administered $2.0 \mathrm{mg} / \mathrm{kg} \mathrm{Nicotine} \mathrm{and}$ $2.0 \mathrm{mg} / \mathrm{kg}$ of Cd. Subsequently, a separate group of animals $(n=5$; control) received normal saline. The total duration of treatment for all groups was 28 days (P28-P56). At P56, the treatment was discontinued, after which the animals were examined in behavioural tests. Nicotine and cadmium increased the metabolism and food intake in the female adolescent mice. This also corresponded to an increase in weight when compared with the control. However, a combined nicotine-cadmium treatment induced a decline in weight of the animals versus the control. Also, nicotine administration increased the motor function, while cadmium and nicotinecadmium treatment caused a decline in motor activity. Both nicotine and cadmium induced a reduction in memory index; however, nicotine-cadmium treatment induced the most significant decrease in nonspatial working memory.
\end{abstract}

\section{Introduction}

Nicotine, a cholinomimetic alkaloid, plays an important role in the neurophysiologic changes which lead to dependence $[1,2]$. Nicotine is the main active ingredient in tobacco smoke that causes and maintains tobacco addiction [3]. Many studies have revealed that nicotine produces tolerance and leads to psychological and physical dependences in adults [4]. Nicotine has been reported to increase 5-hydroxytryptamine (serotonin, 5-HT) release in the cortex, striatum, hippocampus, dorsal raphe nucleus (DRN), hypothalamus, and spinal cord [5-7]. Also nicotine induces the release of several other neurotransmitters, including acetylcholine (ACh), noradrenaline, GABA, and glutamate [4,8-11]. Although the mechanism of nicotine-related tobacco smoke addiction is unclear, it is suspected to involve several chemical pathways, neurotransmitter, and anatomical changes in different brain areas $[4,12,13]$.

The most predominant method of nicotine use is the inhalation of tobacco smoke which consists of nicotine, cadmium, and other alkaloids $[14,15]$. Interestingly, studies have shown that the effect of nicotine administered alone, on behavior, differs from the observed behavioral changes when it was coadministered with cadmium in tobacco smoke $[16,17]$. On its own, cadmium is a long-standing heavy metal present in the tobacco leaf and the environment, with an average concentration of 0.1 and 0.5 parts per million (ppm) [18]. Following prolonged exposure, coupled with its ubiquitous nature, bioaccumulation and toxicity of cadmium increased in the body of smokers $[18,19]$. In addition, exposure 
through inhalation or ingestion of agroproducts (phosphate fertilizers) often amounts to the accumulation of cadmium up to a concentration of $300 \mathrm{mg} / \mathrm{kg}[18,20,21]$.

In smokers, serum level of cadmium and nicotine is usually higher than that observed in nonsmokers [22, 23]. With both having differential signaling pathways, neurotoxicity is a common action of nicotine, cadmium, and their metabolites. Although nicotine and nicotinic receptors are naturally existing modulators in the central nervous system, use of nicotine from tobacco smoke often increases the concentration of nicotine in the blood and brain tissue causing the observed toxicity $[24,25]$. In effect, several neurotransmitters (dopamine, 5HT, acetylcholine, GABA, glutamate, and epinephrine) are upregulated [26, 27], while signaling molecules like prolactin are suspected to be deregulated [28]. Consequently, this translates into behavioral changes and addiction in the animals. As a result of inhalation of nicotine in tobacco smoke, the presence of cadmium in the smoke plays an important role in the behavioral changes observed in prolonged use and addiction [27, 29]. In addition, studies have shown that anatomical modifications and degenerative changes occur in specific brain regions after prolonged tobacco smoke addiction [30]. This has been attributed to the persistent excitotoxicity of cholinergic receptors through nicotine potentiation and cadmium-mediated reactive oxygen species (ROS) production (cadmium inhibits cytochrome a3 and cytochrome C oxidase) $[29,30]$.

It is evident, from previous findings, that $60 \%$ of smokers start before the age of 14 (adolescent) and 90\% show dependence before the onset of adult hood [31]. In addition, females have shown more sensitivity to nicotine use than males and are described as being more vulnerable to nicotine reward than males $[31,32]$. Despite the striking evidence of the involvement of tobacco smoke on behavior and addiction in adolescent female, the exact interaction between nicotine and cadmium in addiction and toxicity is yet to be elucidated. Using behavioral tests, we have studied the effect of nicotine and/or cadmium on weight, motor activity, and memory function in female adolescent mice to demonstrate the role of cadmium in the observed nicotine susceptibility and weight loss seen in adolescent female mice.

\section{Materials and Methods}

2.1. Drugs. Nicotine bitartrate was generously given by Dr. Maria A. Deluca of Department of Biological Science, University of Cagliari, Cagliari, Italy, and cadmium chloride was obtained from Department of Chemical Sciences, Afe Babalola University, Nigeria. The salts were dissolved in saline (Drugfield Pharmaceutical LTD, Nigeria) and doses are expressed as free base.

2.2. Animal Preparations and Treatment. $N=20$ female adolescent mice (P28) were procured form the animals holding facility of Afe Babalola University. The animals were kept under standard laboratory conditions of 12-hour alternating light and dark, controlled temperature, and adequate ventilation. They were fed on standard rat chow containing proteins, carbohydrate, fats, vitamins, minerals, and water ad libitum. The animals weigh between 9 and 12 gm and were separated randomly into four (4) groups of $n=5$ animals each. Nicotine (Nc) was administered subcutaneously $(2 \mathrm{mg} / \mathrm{Kg})$ to a set of $n=5$ animals, while a separate group received cadmium $(\mathrm{Cd}$; Subcutaneous) at a dose of $2 \mathrm{mg} / \mathrm{Kg}[33,34]$. A combined treatment group was set up and was treated with cadmium $(2 \mathrm{mg} / \mathrm{Kg})$ and nicotine $(2 \mathrm{mg} / \mathrm{Kg})(\mathrm{Nc}+\mathrm{Cd})$, while the control (vehicle) received normal saline for the period of the treatment (28 days; chronic administration). The nicotine and/or cadmium were administered between 9 and 10 am on each day of the treatment from P28 to P56. All protocols were approved by Afe Babalola University Animal Care Committee following the IACUC guidelines for animals use in research and education.

2.3. Weight Measurements. The weight of the animals was measured daily for the duration of the experiment. Also, the daily food intake was measured using metabolic specially designed metabolic cages.

2.4. Behavioral Assays. The behavioral tests were carried out in a closed area with proper illumination and sound control. All behavioral test performance was captured using a digital video recorder and analyzed in Any-Maze software. The animals were trained in the testing area 3 days to the actual test and were allowed to acclimatize to the equipment used for the various tests.

2.5. Rotarod Test. The animals were placed on a rotarod to determine the latency of fall for three separate trials $\left(\mathrm{T}_{1}, \mathrm{~T}_{2}\right.$, and $\mathrm{T}_{3}$ ) during which the speed of the rotarod was gradually increased from $4 \mathrm{rpm}$ to $35 \mathrm{rpm}$. The maximum duration allowed for each trial was 3 minutes.

2.6. Novel Object Recognition Test (NOR). This was done to evaluate the nonspatial working memory of the animals. For the first trial $\left(\mathrm{T}_{1}\right)$ animals were placed in a white opaque test area with two identical objects for 5 minutes following which an intertrial time (IT) of 60 minutes was observed. A second trial $\left(\mathrm{T}_{2}\right)$ was administered during which one of the old objects was replaced with a novel object for 5 minutes in the same test area. The performance of the animals was recorded and analyzed to determine the exploration time on old and new objects in $\mathrm{T}_{2}$, respectively. Subsequent analysis involves the plotting of the memory index using the method of Kruk-Słomka et al. [35].

2.7. Y-Maze. This was done to check the spatial working memory of the animals. The rats were placed facing the edge and allowed making their arm decision for total test duration of 4 minutes. The frequencies of alteration between the arms were recorded as plotted to determine the memory index (percentage alteration).

2.8. Elevated Plus Maze (EPM). Using an elevated plus maze, anxiety linked behaviors were assessed in the animals $(n=$ 5 per group). These include the frequency of head dipping 
(HD), open arm duration (OAD), closed arm duration (CAD), closed arm entries (CAE), and open arm entries (OAE). The duration of the test was 4 minutes per mice.

\section{Statistical Analyses}

The recorded video performance was analyzed using AnyMaze software to determine object exploration time (NOR), percentage arm alteration (Y-maze), and latency of fall (rotarod). Open and closed arm parameters were measured for elevated plus maze. The data was plotted in one-way ANOVA (GraphPad Prism Version 6.0) with Bonferroni post-hoc test. Significance was set at ${ }^{*} P<0.05$ and results were expressed as means \pm standard error of the mean (SEM). Spearman's correlation was used to analyze the average weight gained per day and average food intake per day from P28 to P56.

\section{Results}

4.1. Weight Changes. In order to evaluate the effect of nicotine, cadmium, or a combined treatment on the development of the adolescent female rats, we measured the weight, daily, for the duration of the experiment. Consequently, we observed a significant increase in weight of the animals in the cadmium, nicotine, and $\mathrm{Nc}+\mathrm{Cd}$ group when compared with the control $(P<0.05)$. Progression correlation shows that the cadmium and nicotine treated groups recorded the highest increase in weight from P28 to P56 while the combined treatment with nicotine-cadmium recorded a decline from P46 to P56 when compared with the control (Figure 1).

4.2. Daily Food Intake. To elucidate the effect of nicotine and/or cadmium on metabolism (calorie intake) during the adolescent stage of female development, the daily food intake of each group was recorded for the duration of the experiment. Similar to our observations on weight changes, the nicotine treated animals recorded the most significant increase in food intake rate when compared with the control $\left(P<0.01^{* *}\right)$. Interestingly, the cadmium treated animals recorded an increase in food intake $\left(P<0.05^{*}\right)$ but was less significant versus the nicotine treated group. In addition, cotreatment with nicotine-cadmium recorded a steady decline in food intake around P30-P56. This also correlated with the decline in weight observed in this group versus the control around P46 (Figure 2).

\subsection{Memory Function}

4.3.1. Y-Maze. This was used to analyze the spatial working memory in the female adolescent mice after an exposure to nicotine, cadmium, or a combination of both (nicotinecadmium) during the adolescence period (P28-P56). We evaluated the percentage alteration between the arms $\mathrm{A}, \mathrm{B}$, and $\mathrm{C}$ and plotted the memory index (MI). No significant change in spatial memory function was observed when the treated groups were compared with the control (Figure 3).

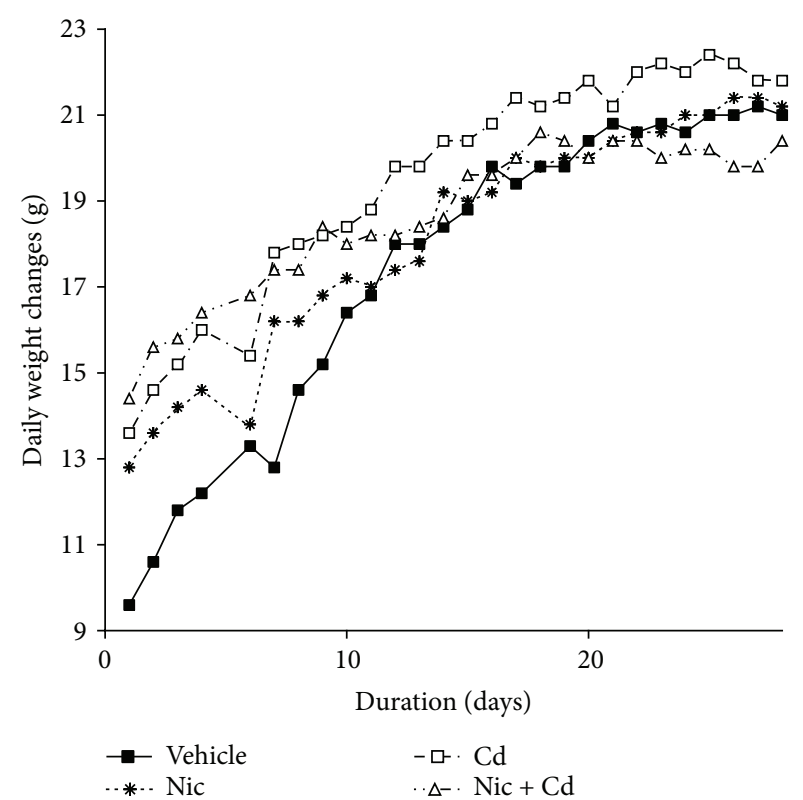

FIGURE 1: Spearman's correlation showing the time-dependent weight changes in the treatment groups and control. The nicotine and/or cadmium treatment caused a significant increase in average weight from P28 to P56 when compared with the control. It was observed that nicotine-cadmium treatment induced a decline in weight around P37 versus saline administered mice statistically. This is suggestive that the weight loss associated with tobacco use is not an exclusive effect of nicotine, but an effect of combined nicotine and cadmium in tobacco smoke.

4.3.2. NOR. The exploration time on old and new objects was determined and used in calculating the memory index. Although no statistically significant decline in memory index was observed in the nicotine treated group, empirical quantification shows a reduction in memory index versus the control. Furthermore, the cadmium treatment and the combined treatment group showed a decline in memory function $(P<0.01)$ (Figure 4$)$.

4.3.3. Rotarod. The latency of fall (LOF) was evaluated for $n=5$ animals in each group. Three (3) trails $\mathrm{T}_{1}, \mathrm{~T}_{2}$, and $\mathrm{T}_{3}$ were conducted for each animal for duration of 3 minutes per trial. An intertrial time (IT) of 12 minutes was also observed between the consecutive tests. The average time spent on the rotarod for the three trials was determined as the representative value for each animal. A significant increase in LOF was observed in the nicotine treated group $(P<0.05)$ when compared with the control. However, cadmium and nicotine-cadmium treatment caused a significant decrease in motor function, recording a decrease in LOF when compared with the control $(P<0.01)$ and the nicotine treatment group $(P<0.001)$. Similar to our findings in memory function, a combination of nicotine and cadmium induced a decline in motor function significantly when compared with nicotine $(P<0.001)$ and cadmium $(P<0.05)$ (Figure 5). 


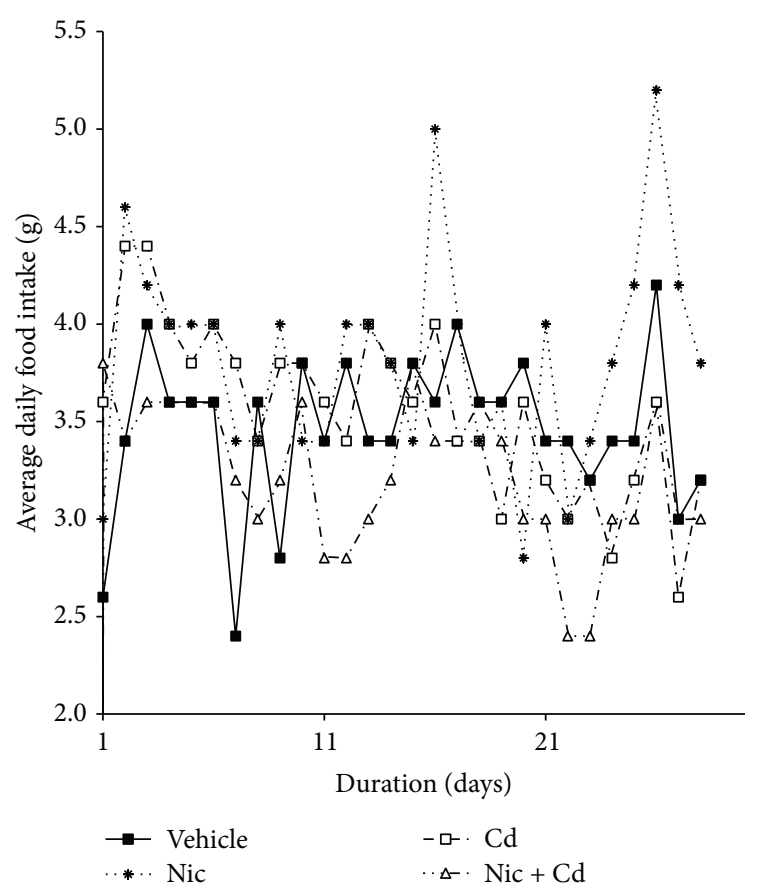

Figure 2: Average daily food intake (P28 to P56) in female adolescent mice. Similar to the observations in weight changes, the average daily food intake increased in the nicotine and cadmium treated groups when compared with the control. Consequent of nicotine-cadmium treatment, a decline in food intake was observed around $\mathrm{P} 46$ versus the saline treated mice. This precedes the weight loss in this group (P50) as shown in Figure 1. Each point represents daily recorded food intake/group expressed as mean \pm SEM.

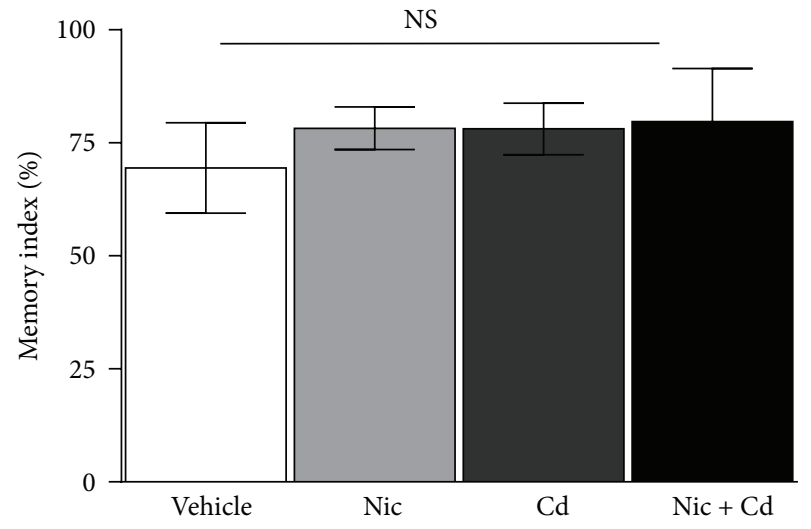

FIGURE 3: Percentage arm alteration expressed as memory index in Y-maze. No statistical significance was observed in spatial memory function for the treatment groups when compared with the control. Empirically, slight increase was seen in all treatments with the highest recorded in the combined nicotine-cadmium treatment.

4.3.4. Elevated Plus Maze (EPM). We employed the behavioral assay technique to detect anxiety related behaviors in the female adolescent mice after nicotine and/or cadmium treatment. There was a statistical significance in the open and closed arm distance for the treatment groups when compared with the control $(P<0.05)$ ( $\uparrow$ anxiety). The nicotine $(\mathrm{Nc})$

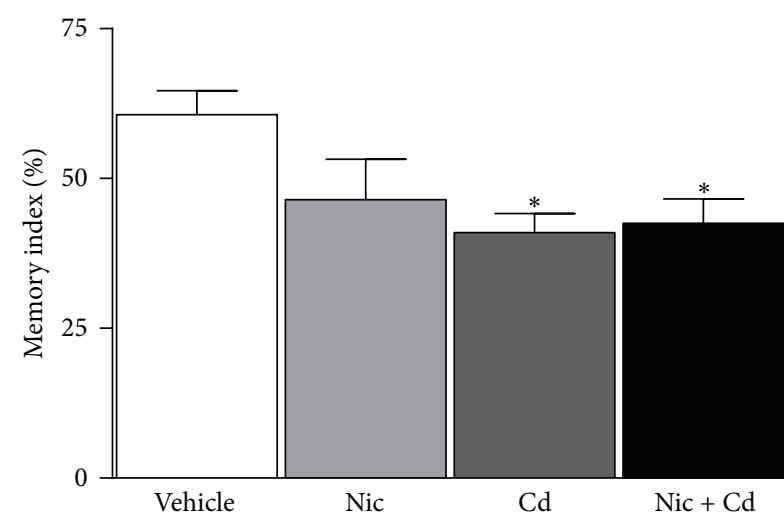

FIgURE 4: Novel object recognition memory index. A significant decline in memory index was observed in the cadmium and combined (nicotine-cadmium) treatment groups $(P<0.05)$ when compared with the control. Although nicotine treatment recorded no statistically significant decline in memory index, empirical quantification shows a decline in the memory index versus the control. However, comparing the nicotine treated group with the combined treatment group demonstrates the effect of cadmium in facilitating decline in memory function when coadministered with nicotine, similar to what is observed in tobacco smoke (statistical significance: $\left.{ }^{*} \mathrm{P}<0.05\right)$.

and cadmium $(\mathrm{Cd})$ treated groups recorded a significant increase in closed arm distance ( $\uparrow$ anxiety) versus the open arm distance for the same group and the control (OA and CA distance) (Figures 6(a) and 6(b)). The combined treatment (nicotine-cadmium) showed a reduction in exploration distance for both open and closed arms (Figure 6(b)); however, it also recorded a more significant closed arm distance versus the open arm distance ( $\uparrow$ anxiety; $P<0.01$ ). We next analyzed the duration spent by each group in either the open or closed arms to further determine the magnitude of anxiety. Similar to our findings in the arm distance analysis, the treatment groups recorded significantly higher durations in the closed arm versus the open arm ( $\uparrow$ anxiety) when compared with the control $(P<0.05)$. Similarly, the most significant increase in CAD was observed in the nicotinecadmium treatment ( $\uparrow$ anxiety) (Figure 6(c)). Analysis of the frequency of open and closed arm entries further supports these findings as the combined treatment (nicotinecadmium) showed more anxiety with the least rate of total arm entries (decreased exploration) (Figure 6(d)).

\section{Discussion}

Taken together our findings suggest that nicotine or cadmium treatment induced an increase in food consumption rate in the female adolescent mice. In addition, a steady increase in weight was observed in these groups when compared with the control. However, the nicotine-cadmium treatment showed a decline in food consumption rate and weight from P37 to P56, suggesting the negative effect of this combination on metabolism and development. Furthermore we observed that nicotine increased motor function in the mice when 


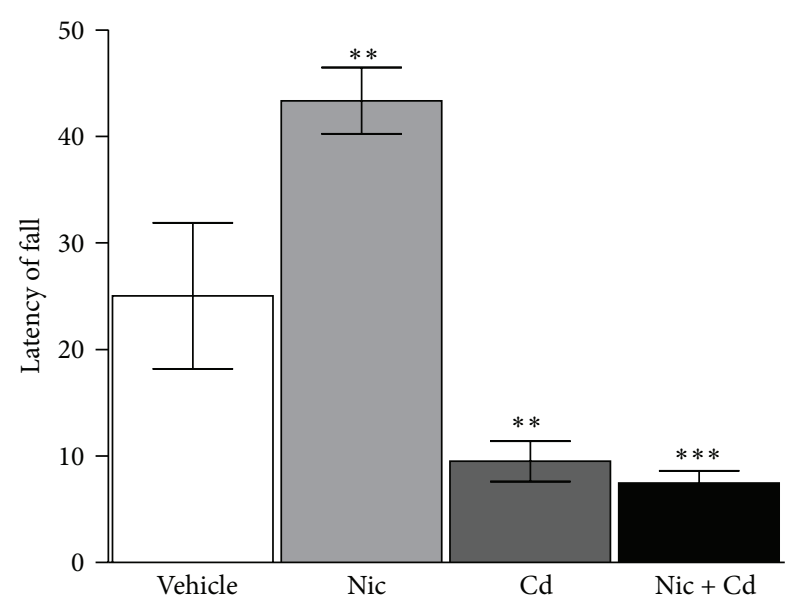

FIGURE 5: Latency of fall (LOF) in rotarod test for motor function. Treatment with nicotine increased the latency of fall significantly ( $\uparrow$ motor function) when compared with the control group $(P<$ 0.05). This is attributed to the role of nicotine in stimulating cholinergic receptors at neuromuscular junctions and increasing epinephrine release. The cadmium $(P<0.01)$ and nicotinecadmium $(P<0.001)$ treated groups showed a significant decrease in motor function $(\downarrow L \mathrm{LOF})$ when compared with the control and nicotine treated group $(P<0.001)$. Subsequent analysis shows that nicotine-cadmium treatment reduced motor function significantly when compared with nicotine $(P<0.001)$ and cadmium $(P<0.05)$ treatment groups, respectively (statistical significance: ${ }^{*} P<0.05$, ${ }^{* *} P<0.01$, and $\left.{ }^{* * *} P<0.001\right)$.

compared with the control while cadmium induced a decline in motor function. However, coadministration of nicotinecadmium induced the most significant decline in motor function versus the control. We have also shown that nicotine administered between P28 and P56 caused no significant decline in memory function for spatial (Y-maze) and nonspatial (NOR) memory while cadmium induced a decline in memory function. Similar to our observations in motor function, a combination of nicotine-cadmium treatment induced the most significant decline in memory function; especially in novel object recognition memory. Taken together we deduce that nicotine, when singly administered, holds the potential of reducing memory function after prolonged use. However, its combination with cadmium induces a significant decline in memory function over a short period of time. Similarly, cadmium administration induced a more significant decline in nonspatial working memory when compared with the nicotine-cadmium and control.

Anxiety linked behaviors were also observed both in the nicotine and treatment groups (Figure 6(b)). In EPM, the nicotine and cadmium treatment groups explored the closed arm of the maze more than the open $\operatorname{arm}(P<0.05)$ when compared with the control. However a combined treatment of nicotine and cadmium caused a decrease in exploration distance in the closed arm of the maze. This also corresponded to an increase in closed arm duration, thus depicting anxiety in this treatment group (reduced exploration distance and prolonged closed arm duration) (Figures 6(a)6(c)). The findings from EPM show that both nicotine and cadmium can induce anxiety-linked behaviors after prolonged use, a combined treatment of nicotine-cadmium, similar to the tobacco smoke, caused a significant increase in anxiety in the female adolescent mice. Other anxiety linked symptoms observed in the combined treatment group (nicotine-cadmium) include an increased food consumption, decreased weight, and a decline in working memory.

Studies have shown that nicotine, a cholinergic agonist, is capable of altering the rate of food consumption and energy expenditure, thus leading to weight changes [36, 37]. Although the exact mechanism remains elusive, both central and peripheral mechanisms are suspected to be involved. The significant effect of nicotine on metabolism has been described to occur through its ability to potentiate several cholinergic receptors in the central nervous system (hypothalamus) and peripheral nervous system [37, 38]. Our findings suggest that nicotine treatment during the adolescence period increased basal metabolism (food intake) and the weight of the animals through nicotine mediated reward system that affected brain energy metabolism regulation [38]. Although nicotine is the major addictive substance in tobacco smoke, our results show that cadmium participates in the change in caloric intake that affect weight and energy expenditure (Figures 1 and 2). Our findings show that nicotine use increased the body weight and calorie intake in female adolescent mice; contrary to the idea that nicotine in tobacco is responsible for the weight loss seen in smokers $[39,40]$. In addition, our results prove that a combination of nicotine and cadmium might be responsible for the weight loss associated with tobacco smoke as shown in Figures 1 and 2 (nicotinecadmium treatment). Thus we deduce from these findings that changes observed in tobacco smoke are not exclusively nicotinic but a combination of cholinergic-signaling and other associated cellular mechanisms involving cadmium.

An increase in metabolism induced by nicotine is often associated with an increased motor function [41]. A model that addresses such function is linked with the ability of nicotine to stimulate nicotine receptors at the neuromuscular junctions and increase the release of epinephrine [42, 43]. As a result, glucose uptake increases in the muscles, thereby facilitating an increase in motor function [44]. Although this feature of nicotine use has been explored in Parkinson's disease (PD) therapeutics, it is often associated with increased anxiety due to the central effect of epinephrine [45]. Similarly, other studies have reported that smokers show a late onset of motor dysfunctions in PD when compared to nonsmokers due to the ability of nicotine to modify dopaminergic $\left(D_{1}\right)$ receptor dynamics following degeneration of dopaminergic neurons in the striatum [46-48]. However, this effect is not exclusive to the effect of nicotine on motor function. Recent findings suggest that nicotine modulates intrasynaptic dopaminergic release as part of its reward system. In addition, the $D_{1}$ receptors observed in the striatum are also upregulated in the reward related brain regions [49].

Although nicotine increased motor function, subsequent analysis of animals treated with both nicotine-cadmium and cadmium showed a decline in motor functions when compared with the control and the nicotine group (Figure 4). The role of cadmium in inhibition of tissue respiration 


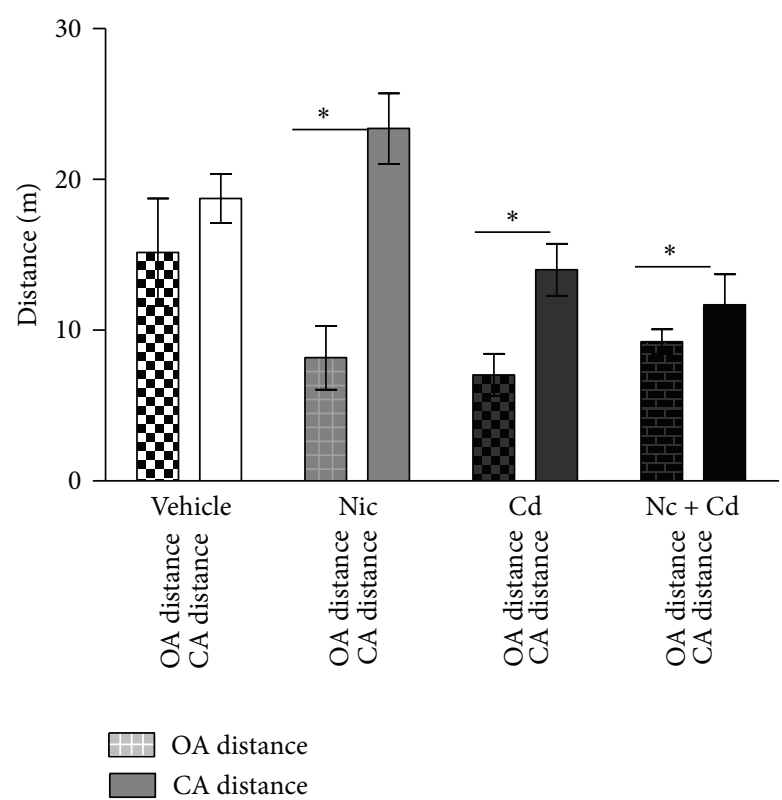

(a)

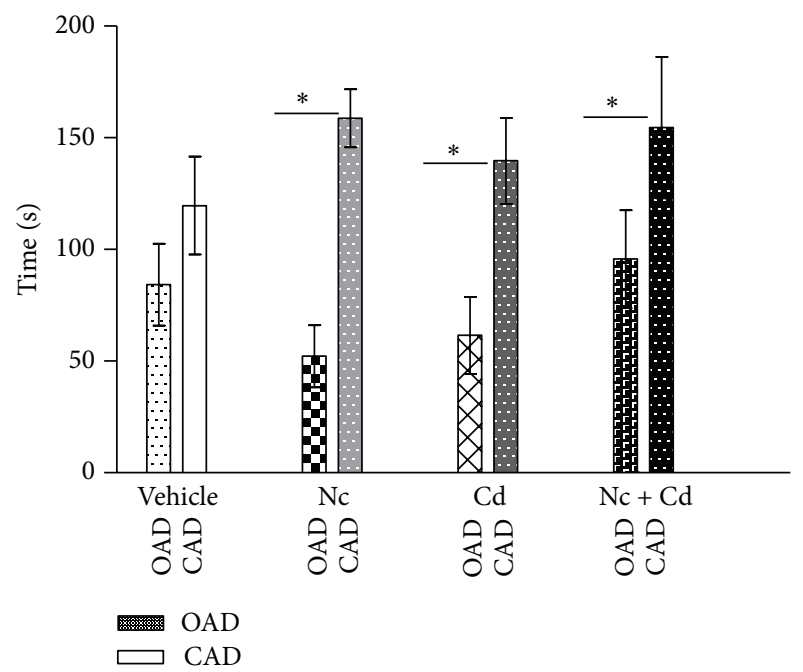

(c)
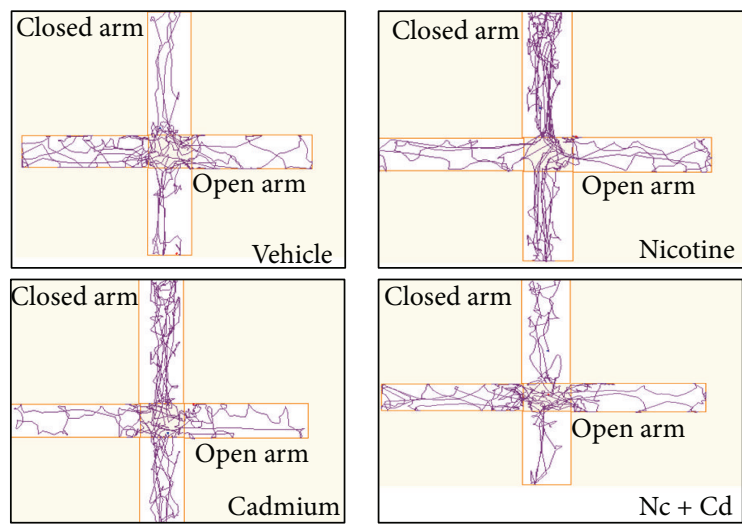

(b)

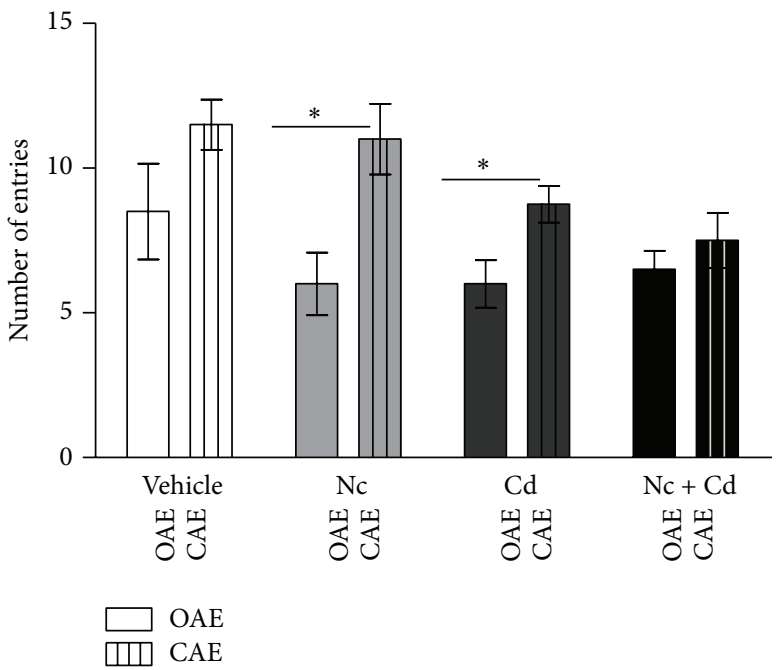

(d)

Figure 6: $(a, b)$ The average distance travelled in meters in elevated plus maze. (a) The open arm distance (checked bars) and closed arm distance (plain bars) for the treatment groups were compared versus the control. Nicotine treated female mice showed a significance in the distance covered in the open arm when compared with the control $(P<0.05)$. In furtherance, cadmium treated mice recorded a decrease in total exploration distance when compared with the nicotine treated group $(P<0.05)$. Anxiety linked behaviors were observed in the nicotine treatment as they explored the closed arm more than the open arm. Also, the cadmium and nicotine-cadmium treated groups showed a significant decline in exploration time and explored the closed arm more than the open arm (statistical significance: $\left.{ }^{*} P<0.05\right)$. (b) Track plots of the mice during the 4 minutes exploration in EPM using Any-Maze software to demonstrate the open and closed arm distances. (c and d) Elevated plus maze open/closed arm entries and duration. (c) Close arm (CAD::stripes) and open arm (OAD::plain) durations for the treatment groups versus the control. Increased CAD was observed in the nicotine, cadmium, and nicotine-cadmium treatment group $(P<0.05)$, thus depicting increased anxiety in these animals. (d) Bar chart showing the frequency of close arm (CAE) and open arm $(\mathrm{OAE})$ entries for the treatment groups and control. A significant difference $(P<0.05)$ was observed between the CAE and OAE in the nicotine and cadmium treated groups $\left({ }^{*} P<0.05\right)$. No significance was seen when the OAE and CAE were compared for the combined nicotine-cadmium treatment group (statistical significance: $\left.{ }^{*} \mathrm{P}<0.05\right)$. 
is suspected to be involved in the inhibition of synaptic function and transmission, thus blocking nicotinic synaptic effects in prolonged tobacco use (Figure 4). Similarly, Bierbower and Cooper demonstrated that carbon dioxide $\left(\mathrm{CO}_{2}\right)$ produced via inhibition of tissue respiration blocks autonomic responses and inhibits glutamate neurotransmission at the neuromuscular junctions. Other studies have shown that cadmium is involved in the blockade of synaptic inputs at neuromuscular junctions and the central nervous system via upregulation of inhibitory neurotransmitter (GABA) and cellular carbon dioxide, thus accounting for its effect in reducing motor function when coadministered with nicotine or solely $[50,51]$.

Primary targets for the central effect of nicotine are receptors involved in the reward system and memory [5254]. Similar to our findings, other experiments involving the post natal exposure of mice to nicotine between P28 and P43 have shown that gender variations exist in the observed memory functions and anxiety linked behaviors. While no significant change was observed in males, female animals showed an increased sensitivity to nicotine induced memory loss and anxiety [55]. Similarly, cadmium induced toxicity is capable of facilitating synaptic blockade and oxidative stress which mediates degenerative changes and memory loss. However, the effects of both nicotine and cadmium in adolescent have been linked with memory loss during use and after withdrawal [56]. Other documented evidence of tobacco-mediated memory loss includes depletion of nicotinic receptors in the prefrontal cortex $[57,58]$, increase in number of immature hippocampal neurons $[59,60]$, and inhibition of synaptic transmission both in the prefrontal cortex and hippocampus $[59,61]$.

5.1. Public Health Significance. Social or societal stress is often associated with adolescent humans who often engage in the abuse of nicotine, cannabinoids, amphetamine, and alcohol $[62,63]$. Other reports have shown that tobacco use is most prevalent among adolescents and young adults between the ages of 13 and 25 years; furthermore, most start to smoke between the ages of 12 and 14 years [64, 65]. Although no significant difference was observed between the male and female adolescent smoking rates [66], females, however, show more susceptibility to nicotine-tobacco reward and addiction [31]. The long-term effect of tobacco use in adolescent females includes addiction $[31,66]$, sexual development [28], attention and memory deficits [67], and dependence during pregnancy [68].

\section{Conclusion}

We conclude that nicotine and cadmium when administered separately induced an increase in metabolism, food intake, and weight in adolescent female mice. However, the decrease in weight associated with tobacco smoke is as a result of nicotine-cadmium synergy rather than just nicotine. Also, nicotine increased motor function while cadmium and a combination of cadmium and nicotine decreased motor function due to synaptic inhibition by cadmium. Nicotine, cadmium, and nicotine-cadmium treatment decreased memory function, induced synaptic blockade, and possibly downregulated nicotinic receptors in the prefrontal cortex and hippocampus.

\section{Conflict of Interests}

The authors hereby declare there is no conflict of interests associated with this study or any of the procedures and materials used for the purpose of the study.

\section{Acknowledgments}

The authors acknowledge Dr. Maria A. Deluca of Department of Biological Science, University of Cagliari, for her generous gift of the nicotine salt and also Mr. Oso of the Department of Chemical Sciences, Afe Babalola University, who worked with us in the preparation of salts and reagents. The authors will also like to appreciate Professor Micaela Morelli of the University of Cagliari for hosting Philip Adeniyi during his visit for the CEAN-ISN fellowship.

\section{References}

[1] B. le Foll and S. R. Goldberg, "Effects of nicotine in experimental animals and humans: an update on addictive properties," in Nicotine Psychopharmacology, vol. 192 of Handbook of Experimental Pharmacology, pp. 335-367, Springer, Berlin, Germany, 2009.

[2] K. A. Perkins, "Improving efficiency of initial tests for efficacy in smoking cessation drug discovery," Expert Opinion on Drug Discovery, vol. 9, no. 11, pp. 1259-1264, 2014.

[3] J. J. Ware and M. R. Munafò, "Determining the causes and consequences of nicotine dependence : emerging genetic research methods," Current Psychiatry Reports, vol. 16, no. 10, p. 477, 2014.

[4] D. Kota, B. R. Martin, S. E. Robinson, and M. I. Damaj, "Nicotine dependence and reward differ between adolescent and adult male mice," Journal of Pharmacology and Experimental Therapeutics, vol. 322, no. 1, pp. 399-407, 2007.

[5] S. Hernandez-Lopez, J. Garduño, and S. Mihailescu, "Nicotinic modulation of serotonergic activity in the dorsal raphe nucleus," Reviews in the Neurosciences, vol. 24, no. 5, pp. 455-469, 2013.

[6] E. R. Polina, V. Contini, M. H. Hutz, and C. H. D. Bau, "The serotonin $2 \mathrm{~A}$ receptor gene in alcohol dependence and tobacco smoking," Drug and Alcohol Dependence, vol. 101, no. 1-2, pp. 128-131, 2009.

[7] E. Shearman, S. Fallon, H. Sershen, and A. Lajtha, "Nicotineinduced monoamine neurotransmitter changes in the brain of young rats," Brain Research Bulletin, vol. 76, no. 6, pp. 626-639, 2008.

[8] S. Singer, S. Rossi, S. Verzosa et al., "Nicotine-induced changes in neurotransmitter levels in brain areas associated with cognitive function," Neurochemical Research, vol. 29, no. 9, pp. 17791792, 2004.

[9] M. J. Neal, J. R. Cunningham, and K. L. Matthews, "Activation of nicotinic receptors on GABAergic amacrine cells in the rabbit 
retina indirectly stimulates dopamine release," Visual Neuroscience, vol. 18, no. 1, pp. 55-64, 2001.

[10] K. Miyasato, "Recent advances in research on nicotine dependence and reward mechanism," Nihon Yakurigaku Zasshi, vol. 117, no. 1, pp. 27-34, 2001.

[11] P. J. Kenny, S. E. File, and M. J. Neal, "Evidence for a complex influence of nicotinic acetylcholine receptors on hippocampal serotonin release," Journal of Neurochemistry, vol. 75, no. 6, pp. 2409-2414, 2000.

[12] P. B. S. Clarke, M. Quik, F. Adlkofer, and K. Thurau, Eds., Effects of Nicotine on Biological Systems II, Advances in Pharmacological Sciences, Birkhäuser, Boston, Mass, USA, 1995.

[13] E. F. Domino, "Tobacco smoking and nicotine neuropsychopharmacology: some future research directions," Neuropsychopharmacology, vol. 18, no. 6, pp. 456-468, 1998.

[14] N. Djulančić, V. Radojičić, and M. Srbinovska, “The influence of tobacco blend composition on carbon monoxide formation in mainstream cigarette smoke," Arhiv za Higijenu Rada $i$ Toksikologiju, vol. 64, no. 1, pp. 107-113, 2013.

[15] T. Streibel, S. Mitschke, T. Adam, and R. Zimmermann, “Timeresolved analysis of the emission of sidestream smoke (SSS) from cigarettes during smoking by photo ionisation/timeof-flight mass spectrometry (PI-TOFMS): towards a better description of environmental tobacco smoke," Analytical and Bioanalytical Chemistry, vol. 405, no. 22, pp. 7071-7082, 2013.

[16] C. R. E. Coggins, W. J. McKinney Jr., and M. J. Oldham, “A comprehensive evaluation of the toxicology of experimental, nonfiltered cigarettes manufactured with different circumferences," Inhalation Toxicology, vol. 25, no. 2, pp. 69-72, 2013.

[17] F. Hammal, A. Chappell, T. C. Wild et al., ““'Herbal” but potentially hazardous: an analysis of the constituents and smoke emissions of tobacco-free waterpipe products and the air quality in the cafés where they are served," Tobacco Control, 2013.

[18] R. A. Bernhoft, "Cadmium toxicity and treatment," The Scientific World Journal, vol. 2013, Article ID 394652, 7 pages, 2013.

[19] C. Gerspacher, U. Scheuber, G. Schiera, P. Proia, D. Gygax, and I. di Liegro, "The effect of cadmium on brain cells in culture," International Journal of Molecular Medicine, vol. 24, no. 3, pp. 311-318, 2009.

[20] Y. Jiao, C. A. Grant, and L. D. Bailey, "Effects of phosphorus and zinc fertilizer on cadmium uptake and distribution in flax and durum wheat," Journal of the Science of Food and Agriculture, vol. 84, no. 8, pp. 777-785, 2004.

[21] C. A. Grant and S. C. Sheppard, "Fertilizer impacts on cadmium availability in agricultural soils and crops," Human and Ecological Risk Assessment, vol. 14, no. 2, pp. 210-228, 2008.

[22] M. J. Jarvis, G. A. Giovino, R. J. O’Connor, L. T. Kozlowski, and J. T. Bernert, "Variation in nicotine intake in U.S. cigarette smokers over the past 25 years: evidence from NHANES surveys," Nicotine \& Tobacco Research, 2014.

[23] I. P. Oyeyipo, Y. Raji, and A. F. Bolarinwa, "Nicotine alters serum antioxidant profile in male albino rats," North American Journal of Medical Sciences, vol. 6, no. 4, pp. 168-171, 2014.

[24] P. Naik, N. Fofaria, S. Prasad et al., "Oxidative and proinflammatory impact of regular and denicotinized cigarettes on blood brain barrier endothelial cells: Is smoking reduced or nicotine-free products really safe?" BMC Neuroscience, vol. 15, article 51, 2014.

[25] V. Prabhakar, G. Jayakrishnan, S. V. Nair, and B. Ranganathan, "Determination of trace metals, moisture, $\mathrm{pH}$ and assessment of potential toxicity of selected smokeless tobacco products,"
Indian Journal of Pharmaceutical Sciences, vol. 75, no. 3, pp. 262269, 2013.

[26] T. L. Sterley, F. M. Howells, and V. A. Russell, "Nicotinestimulated release of $\left[{ }^{3} \mathrm{H}\right]$ norepinephrine is reduced in the hippocampus of an animal model of attention-deficit/hyperactivity disorder, the spontaneously hypertensive rat," Brain Research, vol. 1572, pp. 1-10, 2014.

[27] S. J. Kim, J. M. Sullivan, S. Wang, K. P. Cosgrove, and E. D. Morris, "Voxelwise lp-ntPET for detecting localized, transient dopamine release of unknown timing: Sensitivity Analysis and Application to Cigarette Smoking in the PET Scanner," Human Brain Mapping, vol. 35, no. 9, pp. 4876-4891, 2014.

[28] B. Bahadori, N. D. Riediger, S. M. Farrell, E. Uitz, and M. F. Moghadasian, "Hypothesis: smoking decreases breast feeding duration by suppressing prolactin secretion," Medical Hypotheses, vol. 81, no. 4, pp. 582-586, 2013.

[29] B. Wang and Y. Du, "Cadmium and its neurotoxic effects", Oxidative Medicine and Cellular Longevity, vol. 2013, Article ID 898034, 12 pages, 2013.

[30] A. E. Abdel Moneim, A. A. Bauomy, M. M. Diab, M. T. Shata, E. M. Al-Olayan, and M. F. El-Khadragy, "The protective effect of Physalis peruviana L. against cadmium-induced neurotoxicity in rats," Biological Trace Element Research, vol. 160, no. 3, pp. 392-399, 2014.

[31] J. R. DiFranza, J. A. Savageau, K. Fletcher et al., "Recollections and repercussions of the first inhaled cigarette," Addictive Behaviors, vol. 29, no. 2, pp. 261-272, 2004.

[32] W. Thiangtham, S. Kalampakorn, T. Intharanate, and W. Sornsin, "The life experiences of Thai women and smoking: a phenomenological study," Journal of the Medical Association of Thailand, vol. 96, supplement 5, pp. S55-S63, 2013.

[33] M. Sofuoglu, A. I. Herman, H. Nadim, and P. Jatlow, "Rapid nicotine clearance is associated with greater reward and heart rate increases from intravenous nicotine," Neuropsychopharmacology, vol. 37, no. 6, pp. 1509-1516, 2012.

[34] Z. X. Xi, K. Spiller, and E. L. Gardner, "Mechanism-based medication development for the treatment of nicotine dependence," Acta Pharmacologica Sinica, vol. 30, no. 6, pp. 723-739, 2009.

[35] M. Kruk-Słomka, A. Michalak, B. Budzyńska, and G. Biała, "A comparison of mecamylamine and bupropion effects on memory-related responses induced by nicotine and scopolamine in the novel object recognition test in mice," Pharmacological Reports, vol. 66, no. 4, pp. 638-646, 2014.

[36] K. L. McFadden, M. A. Cornier, and J. R. Tregellas, "The role of alpha-7 nicotinic receptors in food intake behaviors," Frontiers in Psychology, vol. 5, article 553, 2014.

[37] Y.-H. Jo, D. A. Talmage, and L. W. Role, "Nicotinic receptormediated effects on appetite and food intake," Journal of Neurobiology, vol. 53, no. 4, pp. 618-632, 2002.

[38] H. Chen, S. Saad, S. L. Sandow, and P. P. Bertrand, "Cigarette smoking and brain regulation of energy homeostasis," Frontiers in Pharmacology, vol. 3, article 147, 2012.

[39] K. P. Gennuso, K. M. Thraen-Borowski, T. R. Schlam et al., "Smokers' physical activity and weight gain one year after a successful versus unsuccessful quit attempt," Preventive Medicine C, vol. 67, pp. 189-192, 2014.

[40] K. D. Ward, S. Ahn, F. Mzayek et al., "The relationship between waterpipe smoking and body weight: population-based findings from Syria," Nicotine \& Tobacco Research, 2014.

[41] I. Z. Mathews, R. G. Mills, and C. M. McCormick, "Chronic social stress in adolescence influenced both amphetamine 
conditioned place preference and locomotor sensitization," Developmental Psychobiology, vol. 50, no. 5, pp. 451-459, 2008.

[42] F. I. Carroll, A. Z. Muresan, B. E. Blough et al., "Synthesis of 2-(substituted phenyl)-3,5,5-trimethylmorpholine analogues and their effects on monoamine uptake, nicotinic acetylcholine receptor function, and behavioral effects of nicotine," Journal of Medicinal Chemistry, vol. 54, no. 5, pp. 1441-1448, 2011.

[43] L. Azam, U. Maskos, J.-P. Changeux et al., “ $\alpha$-conotoxin BuIA[T5A;P6O]: a novel ligand that discriminates between $\alpha 6 \beta 4$ and $\alpha 6 \beta 2$ nicotinic acetylcholine receptors and blocks nicotine-stimulated norepinephrine release," The FASEB Journal, vol. 24, no. 12, pp. 5113-5123, 2010.

[44] R. A. Ngala, J. F. O’Dowd, C. J. Stocker, M. A. Cawthorne, and J. R. S. Arch, " $\beta 2$-adrenoceptor agonists can both stimulate and inhibit glucose uptake in mouse soleus muscle through ligand-directed signalling," Naunyn-Schmiedeberg's Archives of Pharmacology, vol. 386, no. 9, pp. 761-773, 2013.

[45] C. Aydin, O. Oztan, and C. Isgor, "Nicotine-induced anxietylike behavior in a rat model of the novelty-seeking phenotype is associated with long-lasting neuropeptidergic and neuroplastic adaptations in the amygdala: Effects of the cannabinoid receptor 1 antagonist AM251," Neuropharmacology, vol. 63, no. 8, pp. 1335-1345, 2012.

[46] J.-R. García-Montes, A. Boronat-García, A.-M. López-Colomé, J. Bargas, M. Guerra-Crespo, and R. Drucker-Colín, "Is nicotine protective against Parkinson's disease? An experimental analysis," CNS and Neurological Disorders-Drug Targets, vol. 11, no. 7, pp. 897-906, 2012.

[47] S. R. Laviolette, N. M. Lauzon, S. F. Bishop, N. Sun, and H. Tan, "Dopamine signaling through D1-like versus D2-like receptors in the nucleus accumbens core versus shell differentially modulates nicotine reward sensitivity," Journal of Neuroscience, vol. 28, no. 32, pp. 8025-8033, 2008.

[48] D. Wang, Y. Noda, Y. Zhou et al., "The allosteric potentiation of nicotinic acetylcholine receptors by galantamine ameliorates the cognitive dysfunction in beta amyloid $_{25-35}$ I.c.v.-injected mice: involvement of dopaminergic systems," Neuropsychopharmacology, vol. 32, no. 6, pp. 1261-1271, 2007.

[49] O. Gozen, B. Balkan, E. Yildirim, E. O. Koylu, and S. Pogun, "The epigenetic effect of nicotine on dopamine D1 receptor expression in rat prefrontal cortex," Synapse, vol. 67, no. 9, pp. 545-552, 2013.

[50] S. M. Bierbower and R. L. Cooper, "The mechanistic action of carbon dioxide on a neural circuit and NMJ communication," Journal of Experimental Zoology Part A: Ecological Genetics and Physiology, vol. 319, no. 6, pp. 340-354, 2013.

[51] M. B. MacIver, "Abused inhalants enhance GABA-mediated synaptic inhibition," Neuropsychopharmacology, vol. 34, no. 10, pp. 2296-2304, 2009.

[52] E. Egecioglu, J. A. Engel, and E. Jerlhag, "The glucagon-like peptide 1 analogue Exendin- 4 attenuates the nicotine-induced locomotor stimulation, accumbal dopamine release, conditioned place preference as well as the expression of locomotor sensitization in mice," PLoS ONE, vol. 8, no. 10, Article ID e77284, 2013.

[53] H. D. Mansvelder, M. Mertz, and L. W. Role, "Nicotinic modulation of synaptic transmission and plasticity in corticolimbic circuits," Seminars in Cell and Developmental Biology, vol. 20, no. 4, pp. 432-440, 2009.

[54] D. L. Due, S. A. Huettel, W. G. Hall, and D. C. Rubin, "Activation in mesolimbic and visuospatial neural circuits elicited by smoking cues: evidence from functional magnetic resonance imaging," The American Journal of Psychiatry, vol. 159, no. 6, pp. 954-960, 2002.

[55] B. Mateos, E. Borcel, R. Loriga et al., "Adolescent exposure to nicotine and/or the cannabinoid agonist CP 55,940 induces gender-dependent long-lasting memory impairments and changes in brain nicotinic and $\mathrm{CB}_{1}$ cannabinoid receptors," Journal of Psychopharmacology, vol. 25, no. 12, pp. 1676-1690, 2011.

[56] Y. Abreu-Villaça, A. C. de Carvalho Graça, A. Ribeiro-Carvalho, V. de Freitas Naiff, A. C. Manhães, and C. C. Filgueiras, "Combined exposure to tobacco smoke and ethanol in adolescent mice elicits memory and learning deficits both during exposure and withdrawal," Nicotine and Tobacco Research, vol. 15, no. 7, pp. 1211-1221, 2013.

[57] N. E. Holz, R. Boecker, S. Baumeister et al., "Effect of prenatal exposure to tobacco smoke on inhibitory control: neuroimaging results from a 25-year prospective study," JAMA Psychiatry, vol. 71, pp. 786-796, 2014.

[58] S. L. Chu, D. Xiao, S. K. Wang et al., "Spontaneous brain activity in chronic smokers revealed by fractional amplitude of low frequency fluctuation analysis: a resting state functional magnetic resonance imaging study," Chinese Medical Journal, vol. 127, no. 8, pp. 1504-1509, 2014.

[59] C. Le Magueresse and E. Cherubini, "Presynaptic calcium stores contribute to nicotine-elicited potentiation of evoked synaptic transmission at CA3-CA1 connections in the neonatal rat hippocampus," Hippocampus, vol. 17, no. 4, pp. 316-325, 2007.

[60] A. Cohen, M. T. Soleiman, R. Talia, G. F. Koob, O. George, and C. D. Mandyam, "Extended access nicotine self-administration with periodic deprivation increases immature neurons in the hippocampus," Psychopharmacology, 2014.

[61] L. Dinur-Klein, P. Dannon, A. Hadar et al., "Smoking cessation induced by deep repetitive transcranial magnetic stimulation of the prefrontal and insular cortices: a prospective, randomized controlled trial," Biological Psychiatry, vol. 76, no. 9, pp. 742-749, 2014.

[62] K. Chau, B. Kabuth, and N. Chau, "Gender and family disparities in suicide attempt and role of socioeconomic, school, and health-related difficulties in early adolescence," BioMed Research International, vol. 2014, Article ID 314521, 13 pages, 2014.

[63] D. R. Camenga, G. Kong, D. A. Cavallo et al., "Alternate tobacco product and drug use among adolescents who use electronic cigarettes, cigarettes only, and never smokers," Journal of Adolescent Health, vol. 55, no. 4, pp. 588-591, 2014.

[64] J. Konfino, D. Ferrante, L. Goldberg, R. Caixeta, and K. M. Palipudi, "Centers for Disease Control and Prevention: tobacco use among youths-Argentina, 2007 and 2012," Morbidity and Mortality Weekly Report, vol. 63, no. 27, pp. 588-590, 2007.

[65] I. T. Agaku, O. A. Ayo-Yusuf, C. I. Vardavas, and G. Connolly, "Predictors and patterns of cigarette and smokeless tobacco use among adolescents in 32 countries, 2007-2011," Journal of Adolescent Health, vol. 54, no. 1, pp. 47-53, 2014.

[66] D. C. Malta, M. Oliveira-Campos, R. R. Prado et al., "Psychoactive substance use, family context and mental health among Brazilian adolescents," Revista Brasileira de Epidemiologia, vol. 17, supplement 1, pp. 46-61, 2012.

[67] E. D. Levin, I. Hao, D. A. Burke, M. Cauley, B. J. Hall, and A. H. Rezvani, "Effects of tobacco smoke constituents, anabasine and anatabine, on memory and attention in female rats," Journal of Psychopharmacology, vol. 28, no. 10, pp. 915-922, 2014. 
[68] L. R. Stroud, G. D. Papandonatos, D. Rodriguez et al., "Maternal smoking during pregnancy and infant stress response: test of a prenatal programming hypothesis," Psychoneuroendocrinology, vol. 48, pp. 29-40, 2014. 


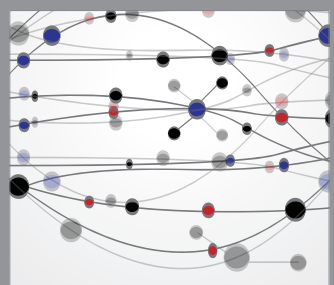

The Scientific World Journal
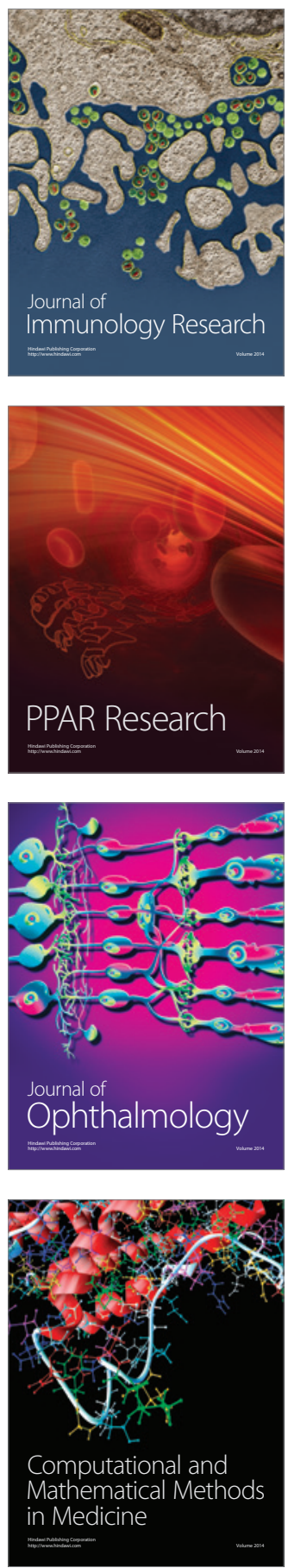

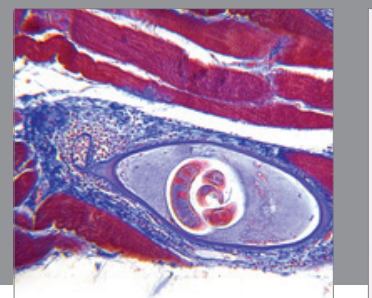

Gastroenterology

Research and Practice
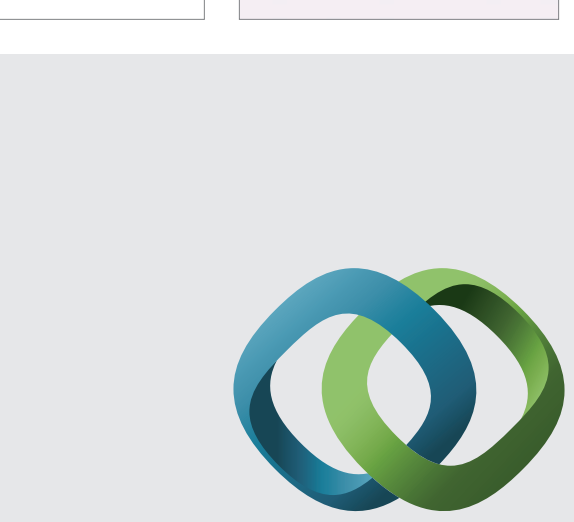

\section{Hindawi}

Submit your manuscripts at

http://www.hindawi.com
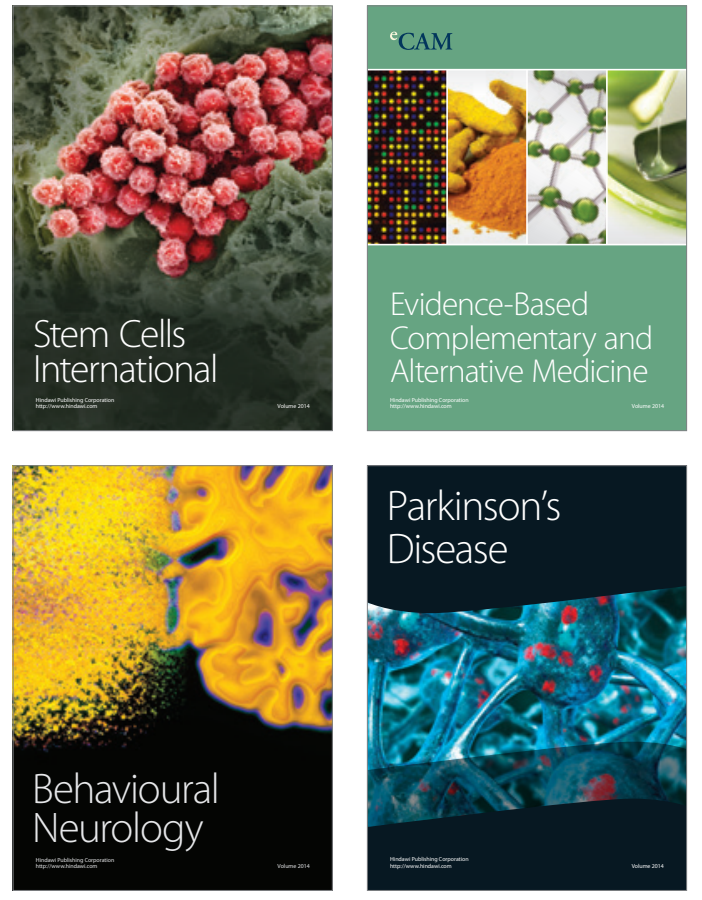
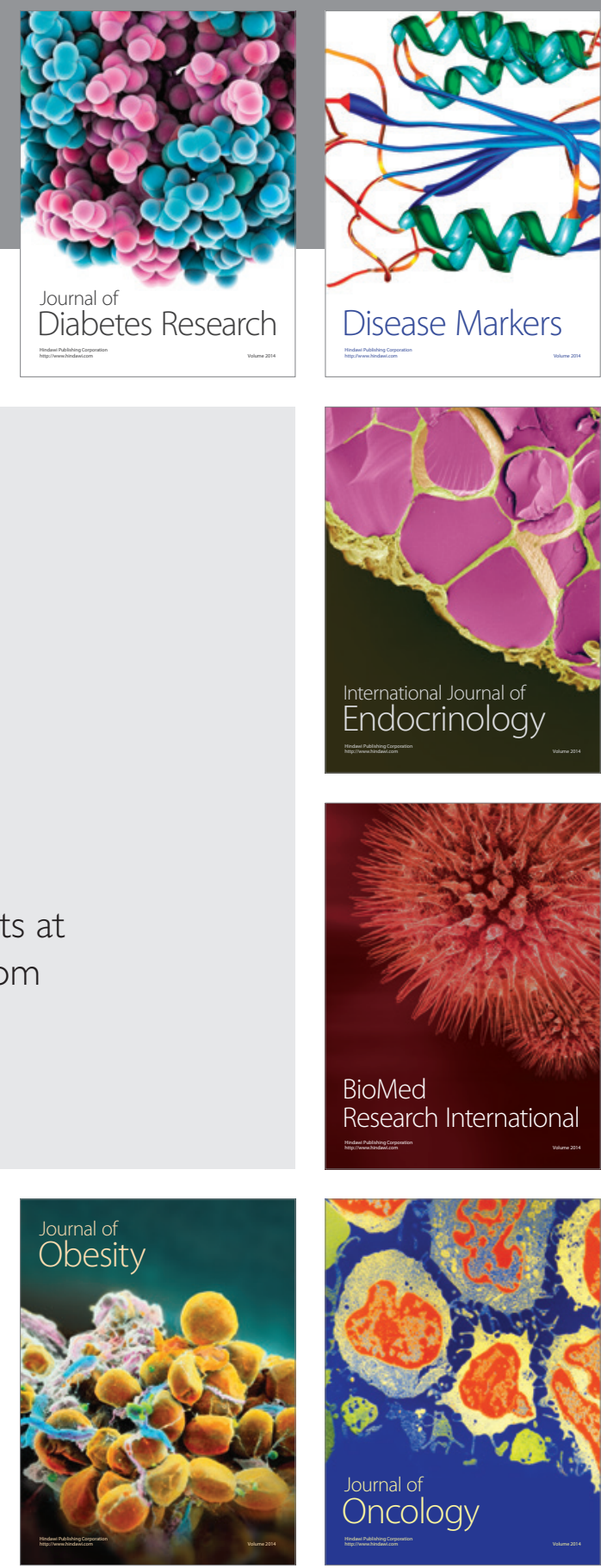

Disease Markers
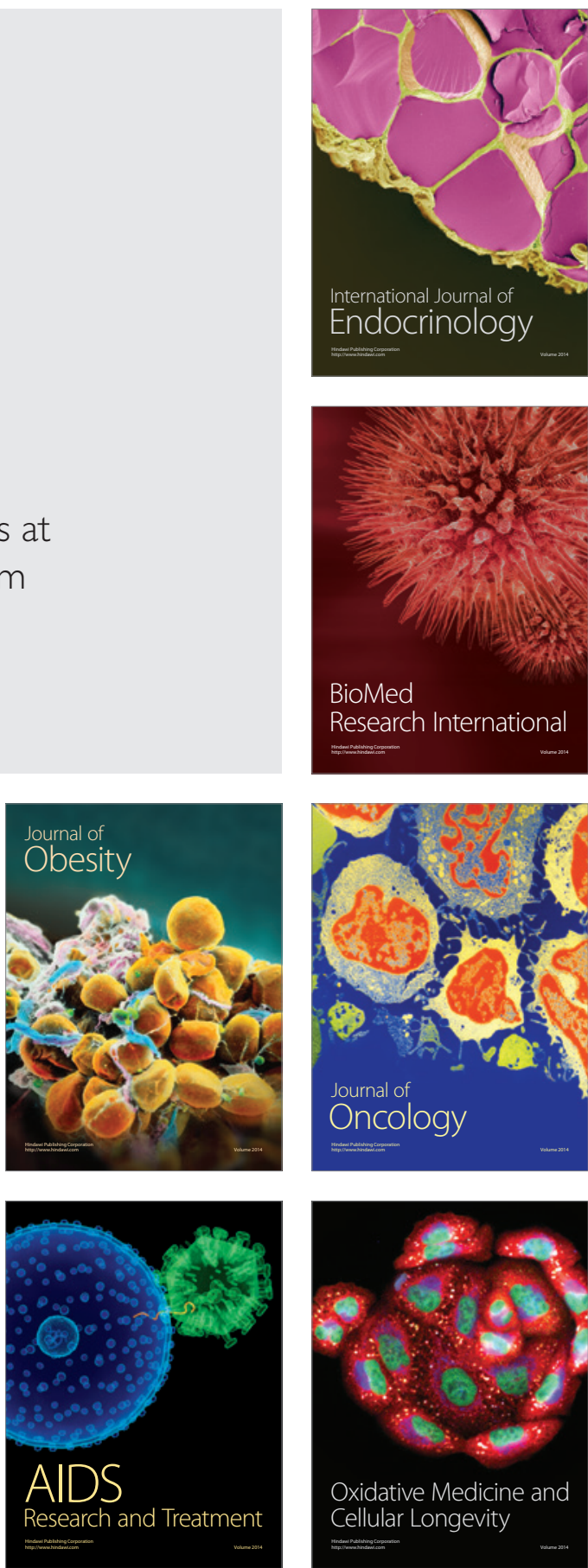